\title{
Médiévales
}

Langues, Textes, Histoire

62 | printemps 2012

Hagiographie et réforme dans l'Occident latin

\section{Stratégies pour la conduite des âmes : la composition poétique et musicale des conductus parisiens au début du XIII ${ }^{\mathrm{e}}$ siècle}

Strategies for the Conduct of Souls : the Poetic and Musical Composition of the Early Thirteenth-Century Parisian Conductus

\section{Anne-Zoé Rillon-Marne}

\section{QpenEdition}

\section{Journals}

Édition électronique

URL : https://journals.openedition.org/medievales/6729

DOI : 10.4000/medievales.6729

ISSN : $1777-5892$

Éditeur

Presses universitaires de Vincennes

Édition imprimée

Date de publication : 2 juin 2012

Pagination : 153-174

ISBN : 978-2-84292-346-4

ISSN : 0751-2708

\section{Référence électronique}

Anne-Zoé Rillon-Marne, « Stratégies pour la conduite des âmes : la composition poétique et musicale des conductus parisiens au début du xIII siècle », Médiévales [En ligne], 62 I printemps 2012, mis en ligne le 05 juillet 2012, consulté le 23 avril 2022. URL : http://journals.openedition.org/medievales/ 6729 ; DOI : https://doi.org/10.4000/medievales.6729 
Médiévales 62, printemps 2012, p. 153-174

\author{
Anne-Zoé RILLON-MARNE
}

\title{
STRATÉGIES POUR LA CONDUITE DES ÂMES : LA COMPOSITION POÉTIQUE ET MUSICALE DES CONDUCTUS PARISIENS AU DÉBUT DU XIII' ${ }^{\mathrm{e}}$ SIÈCLE
}

La musique est certes un art du temps, mais elle peut aussi être un art de l'espace ${ }^{1}$. Dans la théorie musicale médiévale, elle est la représentation terrestre des distances spatiales entre les planètes qui organisent le monde et dont les mouvements ordonnés forment ce que Boèce appelle la musica mundana. Les sons traduisent les nombres et sont l'objet de la science musicale. Les intervalles utilisés pour les mélodies et les harmonies sont donc aussi des données mathématiques dont les mouvements et les modulations sont une manifestation des distances entre les sphères. L'espace architectural, celui de l'église ou de la cathédrale, est lui aussi la représentation terrestre d'un monde céleste. C'est un espace codifié et organisé grâce auquel l'homme peut accéder à la contemplation des vérités supérieures. En tant que praticien, le chanteur fait le lien entre cet espace et le monde des sphères de la théorie musicale. Il réalise concrètement cette harmonie, sans nécessairement en avoir connaissance, cette dernière étant réservée au musicus, celui qui pense la musique. Néanmoins, le cantor remplit l'espace de l'église, ou plutôt anime cet espace symbolique fait de piliers et de voûtes ${ }^{2}$. Sa voix porte un chant organisé de manière à faire vibrer l'air et à habiter ce vide de proportions idéales. Le chant liturgique est d'abord le texte par lequel s'exprime la parole divine. Sa vocalisation pendant l'office permet de le rendre présent, et la mélodie, par ses proportions harmoniques, opère la transcendance entre les cieux et la terre. Les chantres n'ont donc cessé de chercher à améliorer ce chant, de manière à le rendre plus efficace, plus brillant, plus expressif.

1. Une première version de ce texte a été présentée au congrès de l'International Medieval Society of Paris, Space, l'espace au Moyen Âge, Paris, juin 2009.

2. C. Page, «Musicus and Cantor», dans T. Knighton et D. Fallows éd., Companion to Medieval and Renaissance Music, Berkeley-Los Angeles, 1992, p. 74-78. 


\section{La question de l'origine et de la définition du conductus}

Comme d'autres genres musicaux, le conductus $^{3}$ s'est glissé dans les espaces temporels disponibles de la liturgie traditionnelle, tout comme les tropes, les séquences et les hymnes l'ont fait progressivement. C'est là un des moyens d'embellir et d'enrichir les célébrations et de renouveler le répertoire sans toucher à sa base originelle, le plain-chant. Mais, si la place des séquences ou des hymnes dans la liturgie de la messe et des offices semble assez claire, celle des conduits ne répond pas à une logique explicite. Le nom même de conductus n'est pas utilisé de manière univoque par les sources. On le trouve dans des rubriques de manuscrits du XII ${ }^{\mathrm{e}}$ siècle pour introduire des compositions d'apparences diverses. Ce sont généralement des poèmes latins en vers rythmiques accompagnés d'une musique inventée pour eux, mais les structures rencontrées et les propositions de mise en musique sont hétérogènes. Parmi les pièces désignées comme conductus, on rencontre des compositions monodiques ou polyphoniques, de longueurs et d'ambitions variées. Les mots sont soutenus par des dispositifs mélodiques de complexités variables, certains très simples, avec une note par syllabe, tandis que d'autres sont très ornés, allongeant les syllabes par des mélismes.

À l'époque des premières mentions du conduit, le terme versus se confond avec celui de conductus pour désigner des compositions relativement proches par leur forme et leur aspect poétique et musical ${ }^{4}$. Il semble cependant que l'usage de ces deux mots serve à distinguer deux réalités fonctionnelles différentes. L'étymologie du conductus, participe passé du verbe conducere, indique une fonction liée à un mouvement d'ordre spatial ou gestuel ${ }^{5}$. De là, on suppose que le conduit est une pièce destinée à « accompagner» le célébrant pendant son déplacement d'un endroit à un autre. Les rubriques des manuscrits confirment cette interprétation admise par les musicologues ${ }^{6}$. Par exemple, la rubrique du premier conduit de l'Office de la Circoncision de Sens indique conductus ad

3. Dans les sources médiévales, le mot conductus appartient tantôt à la deuxième, tantôt à la quatrième déclinaison. L'usage musicologique est de retenir la quatrième déclinaison. Le pluriel d'usage est donc conductus.

4. M.-D. Popin, «Le versus et son modèle», Revue de musicologie, 73 (1985), p. 19-37.

5. L'origine de l'explication étymologique n'est pas médiévale mais apparait pour la première fois sous la plume de Joseph d'Ortigue (Dictionnaire liturgique, historique et théorique de plainchant, Paris, 1854, article conduis, col. 430-433): «Nous pensons que la signification vraie de ce mot était que le conductus était chanté lorsqu'on reconduisait un personnage soit dans la représentation des mystères, soit dans les cérémonies bizarres tolérées dans l'Église au Moyen Âge.».

6. Les travaux classiques qui tentent de présenter l'évolution du conductus sur l'ensemble de la période médiévale sont L. Ellinwood, «The Conductus», The Musical Quarterly, 27/2 (1941), p. 165-204; J. HandSChIN, «Conductus-Spicilegien», Archiv für Musikwissenschaft, 9/2 (1952), p. 101-119 (cet article s'intéresse avant tout à présenter les sources); J. Knapp, «Conductus », dans S. SADIE éd., The New Grove Dictionary of Music and Musicians, New York, 2001, p. 651-656; 
tabulam. Ailleurs, d'autres rubriques signalent l'arrivée d'un des célébrants à la liturgie ou encore les mouvements des personnages dans le contexte d'un drame liturgique comme le Jeu de Daniel ${ }^{7}$. La fonction du conduit semble être introductive, puisqu'elle comble un espace temporel précédant un événement de la célébration. En outre, les textes de quelques conduits mentionnent, dans leurs premiers ou leurs derniers vers, la lecture qui vient après, permettant de situer précisément la circonstance liturgique de la composition ${ }^{8}$. Dans ce cas, le conduit n'est plus seulement lié à la gestuelle de la célébration, mais aussi à la rhétorique de l'ensemble. La fonction introductive assure la cohérence et la clarté des enchaînements entre les parties. En outre, les relations ponctuelles que l'on peut observer entre le conduit et le Benedicamus Domino ${ }^{9}$ témoignent d'un autre rôle, conclusif cette fois. Dans tous les cas, il s'agit d'un usage d'ordre rhétorique permettant de mieux comprendre les raisons de l'intégration de cette composition nouvelle à la liturgie et de fonder l'explication étymologique. Le conductus est un guide qui clarifie et marque les transitions comme pour «accompagner» l'assemblée dans l'espace-temps du rituel.

Cependant, toutes les pièces des répertoires du XII ${ }^{\mathrm{e}}$ siècle ne correspondent pas à cette définition fonctionnelle. Il arrive que ni la rubrique ni le texte du conduit n'indiquent un quelconque déplacement, déambulation physique et rhétorique entre différents lieux ou moments de la célébration. Ces conduits neutres et sans fonction apparente sont identiques au genre du versus. Les sources entretiennent cette confusion fonctionnelle et générique car les mêmes compositions peuvent apparaître, selon les cas, sous le nom de versus (dans Paris, BnF, lat. 1139 par exemple) ou sous celui de conductus (Offices de Sens et Beauvais déjà mentionnés).

L'explication étymologique du terme conductus est donc relativement instable et fragile lorsque l'on considère ce répertoire dans son ensemble, production poético-musicale qui s'épanouit au cœur du $\mathrm{XII}^{\mathrm{e}}$ siècle à partir de grands centres monastiques du Sud de la France (notamment en Aquitaine à SaintMartial) et, au XIII ${ }^{e}$ siècle, dans les cathédrales du Nord du royaume (Beauvais, Sens). Toutes les sources sont assez concordantes entre elles et pleinement ancrées dans la liturgie où le conductus semble avoir trouvé sa place. Par ailleurs, il existe une autre strate du répertoire des conductus, légèrement plus tardive, assemblée

J. Stevens, Words and Music in the Middle Ages, Song, Narrative, Dance and Drama, 1050-1350, Cambridge, 1986, p. 56-73.

7. Manuscrit LoA (London, British Library, Egerton 2615). Par exemple, la rubrique indique Conductus Danielis venientis ad Regem pour introduire le conduit Hic verus Dei famulus (f.99).

8. W. ArLt, Ein Festoffizium des Mittelalters aus Beauvais, Cologne, 1970, p. 206-208. Voir par exemple le conduit Lector lege hoc de rege, attribué à Fulbert de Chartres dans le Codex Calixtinus.

9. Voir encore le Codex Calixtinus et l'exemple donné par F. Harrison, «Benedicamus, Conductus, Carol : a Newly-Discovered Source», Acta Musicologica, 37 (1965), p. 40. 
de manière plus systématique dans les sources d'origine parisienne composant ce que les musicologues appellent souvent l' «école de Notre-Dame ${ }^{10}$. Pour ces compositions, le lien entre l'explication étymologique et les pièces assemblées comme étant des conduits est encore plus discutable que pour les répertoires et les sources évoquées précédemment. Beaucoup de ces conduits traitent de thèmes qui ne sont liés à aucune fête liturgique, voire incompatibles avec les célébrations ${ }^{11}$. C'est à cette part abondante et diversifiée du répertoire que nous souhaitons réfléchir dans les pages qui suivent. Nous nous demanderons dans quelle mesure le terme de conductus est toujours pertinent pour désigner ce répertoire lyrique latin.

\section{Terminologie du conductus dans le répertoire de Notre-Dame}

Les sources musicales du XIII ${ }^{\mathrm{e}}$ siècle transmettent le répertoire des organa, motets et conduits élaborés pour la plupart à Paris, sous forme de collections qui utilisent l'aspect et la texture musicale des compositions comme outil de classification. Le manuscrit de Florence, le plus important par le nombre des pièces collectées, consacre chacun de ses fascicules aux différents genres musicaux et les classe par affinités et par le nombre des voix. Ainsi, les conduits sont reportés dans les fascicules 6 et 7 pour les compositions polyphoniques et dans les fascicules

10. Il s'agit des deux célèbres manuscrits W1 (Wolfenbüttel, Herzog August Bibliothek, Cod. Guelf. 628 Helmstad.) et W2 (Wolfenbüttel, Herzog August Bibliothek, Cod. Guelf. 1099 Helmstad.) ainsi que du manuscrit F (Firenze, Biblioteca Laurenziana, Pluteus 29.1) et, dans une moindre mesure, de celui de Madrid (Biblioteca Nacional, 20486). Ces sources sont considérées comme «centrales» car de nombreuses compositions sont concordantes et forment un répertoire cohérent désigné sous le terme d' "école de Notre Dame». Bien qu'encore couramment utilisée pour désigner la musique du début du XIII ${ }^{e}$ siècle, cette expression est critiquable. Le répertoire ne présente pas d'élément qui permette de l'interpréter comme une école esthétique au sens moderne du terme. De plus, la cathédrale parisienne n'en est pas le centre exclusif (voir N. LossefF, The Best Concord: Polyphonic Music in Thirteenth-Century Britain, New York - Londres, 1994). Les principales études consacrées aux conduits de Notre-Dame sont: E. GRÖNINGER, Repertoire-Untersuchungen zum mehrstimmigen Notre-Dame Conductus, Regensbourg, 1939 et R. Falck, The Notre-Dame Conductus : A Study of the Repertory, Henryville - Ottawa - Binningen, 1981. Il existe plusieurs éditions musicales. La plus complète est celle de G. A. Anderson (Notre-Dame and Related Conductus, Opera Omnia, 11 vol., Henryville, 1981) bien que les choix de transcription rythmique soient contestables.

11. Il existe aussi des pièces manifestement conçues pour la liturgie. Le meilleur exemple est Salvatoris hodie qui ouvre le sixième fascicule de F (f. 201). Il est aussi noté dans LoA dans lequel la rubrique indique qu'il doit être chanté avant la lecture de l'Évangile lors de la messe de la fête de la Circoncision (W. ArLt, Ein Festoffizium...). Pour d'autres exemples, voir L. Schrade, «Political Compositions in French Music of the 12th and 13th Centuries », Annales musicologiques, Moyen Âge et Renaissance, 1 (1953), p. 9-63. 
10 et 11 pour les compositions monodiques ${ }^{12}$. L'architecte du manuscrit semble avoir eu l'intention de rendre compte et d'organiser l'ensemble de la production musicale liée à Paris ${ }^{13}$. Les volumen consacrés aux organa suivent une logique liturgique mais celle-ci est abandonnée pour les parties concernant les conduits et les motets ${ }^{14}$. On pourrait utiliser le terme de «somme» musicale pour désigner un manuscrit aussi monumental et méthodique. Cependant, aucune rubrique ne mentionne les noms génériques (organum, clausule, motet, conductus) des compositions assemblées.

Pour savoir comment étaient nommées les pièces, il faut faire appel à d'autres sources, notamment les théoriciens de la musique. Le témoignage d'un étudiant anglais est particulièrement utile parce qu'il décrit les pratiques musicales qui ont fait la gloire de Notre-Dame depuis la fin du siècle précédent, à l'usage des notateurs inexpérimentés ${ }^{15}$. Il mentionne un certain Pérotin et lui attribue les incipits de sept compositions dont trois conduits, ainsi que le recueil dans lequel ils ont été conservés, le Magnus liber organi ${ }^{16}$. Les exemples cités et la description du livre concordent avec les sources musicales de Notre-Dame et permettent de reporter la terminologie appliquée au classement des manuscrits qui nous parviennent. Cependant, la réflexion de ces théoriciens s'applique d'abord à la notation de la musique mesurée. Jean de Garlande (homonyme du grammairien)

12. Le dernier fascicule ne comporte que des compositions monodiques latines avec des formes à refrains (rondelli). Leur appartenance au répertoire des conduits peut poser question, bien que leur rapport à la liturgie soit plus vraisemblable que pour les pièces du cahier précédent, en raison des thèmes abordés. Voir R. FALCK, «Rondellus, Canon, and Related Type before 1300 », Journal of the American Musicological Society, 25 (1972), p. 38-57.

13. En regardant dans le détail, on prend conscience d'une réalité beaucoup plus complexe. Certaines pièces sont en effet des «genres» intermédiaires et posent des problèmes de classification. L'usage des termes par les théoriciens contemporains de ces sources montre à quel point le vocabulaire est fluctuant. L'étude la plus récente sur ce manuscrit est celle de B. HaGGH et M. Huglo, «Magnus liber - Maius munus, origine et destinée du manuscrit F», Revue de musicologie, 90/2 (2004), p. 193230 .

14. Voir la présentation d'E. Roesner pour l'édition microfiches du manuscrit (Antiphonarium seu Magnus liber organi et antiphonario : color microfiche ed of the ms., Firenze, Biblioteca Medieca Laurenziana, Pluteus 29.1, Munich, 1996).

15. Il est connu des musicologues sous le nom d'Anonyme IV, depuis l'édition monumentale d'E. de Coussemaker (Scriptorum de Musica Medii aevi, 1864-1876). Pour une édition récente du traité, se reporter à F. ReCKow, Der Musiktraktat des Anonymus 4, Wiesbaden, 1967, 2 vol.; J. HAINES, «Anonymous IV as an Informant on the Craft of Music Writing», The Journal of Musicology, 23/3 (2006), p. 375-425.

16. «Ipse vero magister Perotinus fecit quadrupla optima sicut Viderunt, Sederunt cum habundantia colorum armonicae artis; similiter et tripla plurima nobilissima sicut Alleluia Posui adiutorium, Nativitas etc. Fecit etiam triplices conductus ut Salvatoris hodie et duplices conductus sicut Dum sigillum summi patris ac etiam simplices conductus cum pluribus aliis sicut Beata viscera, etc.» (éd. F. ReCKOw, vol. I, p. 46). 
ou, un peu plus tard, Francon de Cologne proposent une classification des procédés de composition de la polyphonie, mais leur intention est une rationalisation de la pratique écrite, et non une réflexion globale sur les genres, notion indistincte au XIII ${ }^{e}$ siècle. La terminologie leur pose problème et il leur arrive de souligner la difficulté de la polysémie de l'organum ${ }^{17}$. Rien de tel à propos du conduit qui est décrit comme une espèce de polyphonie caractérisée par son style note contre note (déchant) et par l'absence d'emprunt mélodique pour la voix inférieure (cantus prius factus, teneur) ${ }^{18}$. Le conduit monodique est plus rarement évoqué ${ }^{19}$ et ses liens avec la pratique polyphonique ne sont pas explicites. L'application des règles modales de la notation mesurée à ces compositions est une autre source de confusion, car il existe des conduits strictement syllabiques et d'autres qui sont allongés de parties mélismatiques appelées caudae. En effet, la présence ou l'absence de texte est un critère déterminant pour l'application du rythme modal dans la notation carrée du milieu du siècle ${ }^{20}$. De plus, ces écrits théoriques sont postérieurs aux sources musicales, et plus encore à la pratique en question. Les conduits cités et la pratique que ces théoriciens ont à l'esprit ont probablement évolué depuis leur invention et leurs premières interprétations une cinquantaine d'années auparavant. Ces traités sont pourtant les seules approches raisonnées du conduit qui nous parviennent et les seules permettant de construire une description de cette pratique ancrée dans la vie musicale du XIII ${ }^{\mathrm{e}}$ siècle.

17. Par exemple chez l'anonyme IV: «Sciendum, quod organum verbum aequivocum est.» (éd. F. RecKow, vol. I, p. 70).

18. Voici par exemple la description donnée par l'auteur anonyme du Discantus positio vulgaris: «Conductus autem est super unum metrum multiplex consonans cantus, qui etiam secundarias recipit consonantias» (cité par JérôMe de Moravie, Tractatus de musica, éd. S. M. Cserba, Regensbourg, 1935, vol. II, p. 193).

19. Il est cité par l'Anonyme IV qui donne le titre de Beata viscera comme exemple de conductus simplex et par Jean de Garlande qui lui associe la figure rhétorique de la florificatio vocis. La présence d'une voix unique crée également un flou terminologique avec d'autres types de chansons comme la cantilène ou le cantus coronatus chez Johanes de Grocheo.

20. Cette question a longtemps divisé (et divise encore) les musicologues, suscitant une abondante littérature dont J. HANDSCHIN, «Zur Frage der Conductus-Rhythmik», Acta Musicologica, 24/3-4 (1952), p. 113-130; G. A. ANDERSON, «Mode and Change of Mode in Notre-Dame Conductus », Acta Musicologica, 40 (1968), p. 92-114, F. FlindelL, «Conductus in the Later Ars Antiqua», dans In Memoriam Gordon A. Anderson, Henryville - Ottawa - Binnigen, 1984, vol. I, p. 131-210 ; J. KNAPP, «Musical Declamation and Poetic Rhythm in an Early Layer of Notre-Dame Conductus », Journal of the American Musicological Society, 37 (1979), p. 382-407; E. SANDERs, «Conductus and Modal Rhythm», Journal of the American Musicological Society, 38 (1985), p. 439-469; M. E. FASSLER, «Accent, Meter and Rhythm in Medieval Treatises “De rithmis" », Journal of Musicology, 5 (1987), p. 164-190 ; E. SANDERS, «Rithmus», dans Essays on Medieval Music : in Honor of David G. Hugues, Cambridge, 1995, p. 415-440; C. PAGE, Latin Poetry and Conductus Rhythm in Medieval France, Londres, 1997. 
En langue vernaculaire, le flou lexical est le même à propos de la pratique musicale. Conduits et motets sont parfois cités pour désigner la création musicale savante mais les éléments qui les distinguent en tant que genres définis ne sont pas évoqués. Il arrive aussi que les deux termes soient interchangeables. Par exemple, Henri d'Andeli dans son Dit du chancelier Philippe utilise le terme «conduit» pour parler du motet Agmina militie:

Un condut ou il ne faut rien

Fist : Agmina milicie

Que li cler n'ont mie obliée ${ }^{21}$.

Qu'il soit utilisé dans un contexte théorique ou moins spéculatif, le terme semble avoir perdu son sens initial et son rapport étymologique au déplacement dans la liturgie. Pourquoi avoir conservé cet usage terminologique, alors qu'il n'était plus justifié par sa pertinence fonctionnelle? Les différentes couches historiques du répertoire ne présentent pas assez de pièces communes pour expliquer la transmission d'un lexique déjà peu rigoureux dans son usage ancien. On pourrait certes accepter une simple perte de la précision sémantique, comme une banalisation par l'usage. Comment expliquer la permanence du terme conductus dans les sources théoriques et la disparition du versus pourtant plus explicite? Il me semble que les théoriciens et les chanteurs qui utilisent ce terme au XIII ${ }^{\mathrm{e}}$ siècle le font en connaissance de cause, en souvenir des pratiques antérieures, mais aussi parce qu'il fait encore sens pour eux.

Certains musicologues ont déjà cherché à remettre en question l'explication étymologique admise jusqu'alors et généralisée à l'ensemble des répertoires. Bryan Gillingham, dans un article paru en 1991, signale que le verbe conducere se traduit plus volontiers par «mettre ensemble» (joining together) ou encore «contracter», le substantif conductus étant en effet utilisé pour désigner une contraction du corps ${ }^{22}$. L'auteur n'entend pas nier que les traductions de conducere par des verbes de déplacement tels que «conduire», «guider» ou «escorter» soient justes, mais elles lui semblent insuffisantes pour décrire la réalité complexe des compositions liturgiques du XII siècle. Suite à ces remarques, il propose d'interpréter la naissance du conduit comme le résultat d'une «contraction», d'une diminution des compositions binaires et longues que

21. Les Dits d'Henri d'Andeli, éd. A. Corbellari, Paris, 2003, vers 176-178, p. 96. Le motet Agmina militie est issu d'une clausule. Il est conservé dans le manuscrit F (f. 396v) sous forme d'un motet à un seul texte pour les deux voix supérieures et sous forme polytextuelle dans les deux livres de motets, le manuscrit de Bamberg (f. 4) et le manuscrit de La Clayette (f. 377).

22. B. Gillingham, «A New Etiology and Etymology for the Conductus», The Musical Quarterly, 75 (1991), p. 59-73. 
sont les séquences. Le goût pour ces formes diminuées aurait par la suite apporté de plus grandes possibilités de créations et expliquerait le succès du genre ${ }^{23}$.

Cette proposition ne semble pas tenir compte de différences fondamentales entre les deux pratiques du conductus et de la séquence, dans la manière de générer la mélodie. La séquence est élaborée à partir de phrases mélodiques issues de la liturgie, qui deviennent au $\mathrm{XII}^{\mathrm{e}}$ siècle de véritables timbres connus et réemployés pour des textes différents ${ }^{24}$. La composition des conduits présente en général une part de liberté plus grande, si l'on excepte la pratique du contrafactum qui consiste à reprendre intégralement la mélodie d'une composition antérieure. De plus, l'hypothèse porte sur la genèse du conductus et ne cherche pas à expliquer les raisons de la conservation du nom au XIII ${ }^{e}$ siècle. Dans les conduits tardifs du manuscrit de Florence, on rencontre effectivement certaines combinaisons fort complexes de strophes irrégulières simples, doubles et même triples, bien plus proches du lai profane que de la simplicité mélodique de la séquence liturgique. Loin d'être une «réduction» de la pratique de la séquence, de telles ouvres s'en distinguent par une recherche exubérante de subtilité formelle. Comment expliquer que l'on ait continué à utiliser un terme impliquant l'idée de réduction pour des constructions aussi complexes?

C'est probablement dans les œuvres elles-mêmes que des réponses peuvent être trouvées. Les thèmes abordés dans les conduits tardifs des sources parisiennes sont relativement peu variés. Pour simplifier, le répertoire des conduits du XIII ${ }^{e}$ siècle se partage en trois thèmes: le premier relève de la chanson de louange religieuse ou de dévotion (à la Vierge, au Christ ou aux Saints), catégorie qui est la plus proche des répertoires liturgiques antérieurs, bien que l'on ne connaisse pas précisément les circonstances d'utilisation de ces chants dans la liturgie, à Notre-Dame comme ailleurs. Une autre partie des conduits commente ou célèbre des faits historiques. Le fait qu'ils soient datables permet d'envisager le corpus sous un angle chronologique ${ }^{25}$. Enfin, de nombreux textes

23. Ibid., p. 66-69: «In view of the frequent generic interpenetration characteristic of the Aquitanian repertory and the enormous popularity of hymns and sequences prior to the twelfth century, I reiterate that the conductus was initially a hybrid of the two in which a single group of singers (choir or soloists) "joined together" instead of alternating as required in the sequence. The notion that the conductus was first a type of "compressed" or abridged sequence, or a strophic form based on sequence poetry, is supported by the music and is not contradicted by the therorists. Rather than being etymologically drawn from the tenuous and obscure processional connotations of the verb conducere, the term conductus surely reflect the primary meaning of the verb ("to bring together", "join together", or "unite"), and its participial substantive ("a contraction")».

24. Voir notamment les relevés d'E. Misset et P. Aubry, Les Proses d'Adam de Saint-Victor, texte et musique, Paris, 1900 et M. FASLER, Gothic Song: Victorine Sequences and Augustinian Reform in Twelfth-Century Paris, Cambridge, 1993.

25. E. SANDERS, «Style and Technique in Datable Polyphonic Notre-Dame Conductus », dans In Memoriam Gordon A. Anderson, vol. II, p. 505-530; T. B. PAYNE, «Datable Notre-Dame Conductus : 
sont consacrés à la moralisation: dénonciation des mœurs dissolues de l'homme, de la victoire du corps sur l'âme ou observation acerbe du comportement des clercs. Ce dernier thème est relativement novateur puisqu'il emprunte à une tradition poétique revendicatrice en lui ajoutant le soutien mélodique. Dans tous les cas, si «conduite» il y a, il s'agit d'un accompagnement, d'un encadrement qui relie l'auditif au spirituel.

Choisissons deux exemples empruntés au manuscrit de Florence ${ }^{26}$. Homo natus ad laborem (f. 415) et Austro terris influente (f. 299-300v) sont deux conductus attribués à Philippe le Chancelier ${ }^{27}$. Ces deux pièces tiennent une place de choix dans le manuscrit car elles sont insérées à des moments importants de son architecture, en ouverture de fascicules. En effet, les premières pages de chaque cahier se distinguent visuellement par une lettrine richement ornée illustrant l'œuvre choisie pour figurer en exergue ${ }^{28}$. Les concepteurs du manuscrit ont probablement considéré ces pièces comme des modèles représentatifs des compositions qu'elles introduisent ou, pour le moins, comme de très bonnes productions.

\section{Homo natus ad laborem, la voix de l'âme agonisante}

Au début du dixième fascicule consacré aux conduits monodiques - la plus grande collection connue pour la lyrique latine -, le conduit Homo natus ad laborem commence par une citation du Livre de Job $(5,7)$ : homo ad laborem nascitur et avis ad volatum. La lettrine (figure 1) présente un homme travaillant la terre et une femme filant la laine, surmontés d'un oiseau aux ailes déployées

New Observations on Style and Technique», Current Musicology, 64 (2001), p. 104-151.

26. L'édition des textes est donnée en annexes. Les éditions musicales accessibles sont, pour Homo natus ad laborem: G. A. Anderson, Notre-Dame and Related Conductus, Opera Omnia, vol. VI, Henryville, 1981 ou B. Gillingham, Secular Medieval Latin Song : An Anthology, Ottawa, 1993 et pour Austro terris, G. A. Anderson, Notre-Dame..., vol. III et H. Tischler, The Earliest Polyphonic Art Music, vol. I, Ottawa, 2005.

27. Homo natus ad laborem est intégré à une collection de poèmes dont la source, Darmstadt 2777, porte le nom du chancelier parisien. Les arguments en faveur de l'attribution de Austro terris influente sont musicologiques: la voix inférieure du mélisme final de ce conduit a été pourvue d'un nouveau texte (Minor natu filius, lui aussi de Philippe le Chancelier) qui a connu une diffusion indépendante en tant que conduit monodique ( $\mathrm{ms} \mathrm{F}$, f. 450v). Seuls deux autres conduits polyphoniques ont fait l'objet d'un tel traitement et ils sont attribués par les sources à Philippe le Chancelier. L'identité du procédé incite à attribuer l'œuvre au même auteur. Pour ces questions d'attribution, voir P. Dronke, «The Lyrical Compositions of Philip the Chancellor», Studi Medievali, 28 (1987), p. 563-592, reproduit dans Latin and Vernacular Poets of the Middle Ages, Hampshire, 1991 et surtout T. B. Payne, Poetry, Politics, and Polyphony: Philip the Chancellor's Contribution to the Music of Notre Dame School, Ph.D. Diss., Université de Chicago, 1991.

28. Ce codex est orné de quatorze enluminures: une au début de chacun des onze fascicules. Seul le septième fascicule, particulièrement long, est sous-divisé en quatre, au moyen de trois illustrations supplémentaires. 
dans la partie supérieure de la lettre $\mathrm{H}^{29}$. Chacun, qu'il soit homme ou oiseau, y est à sa place et suit le destin voulu par Dieu. Le choix de l'illustration porte donc sur l'incipit du conduit, et non sur l'intégralité de la pièce qui dénonce les déviances de l'homme face à cet ordre établi.

Figure 1: manuscrit F, f. 415 (détail)

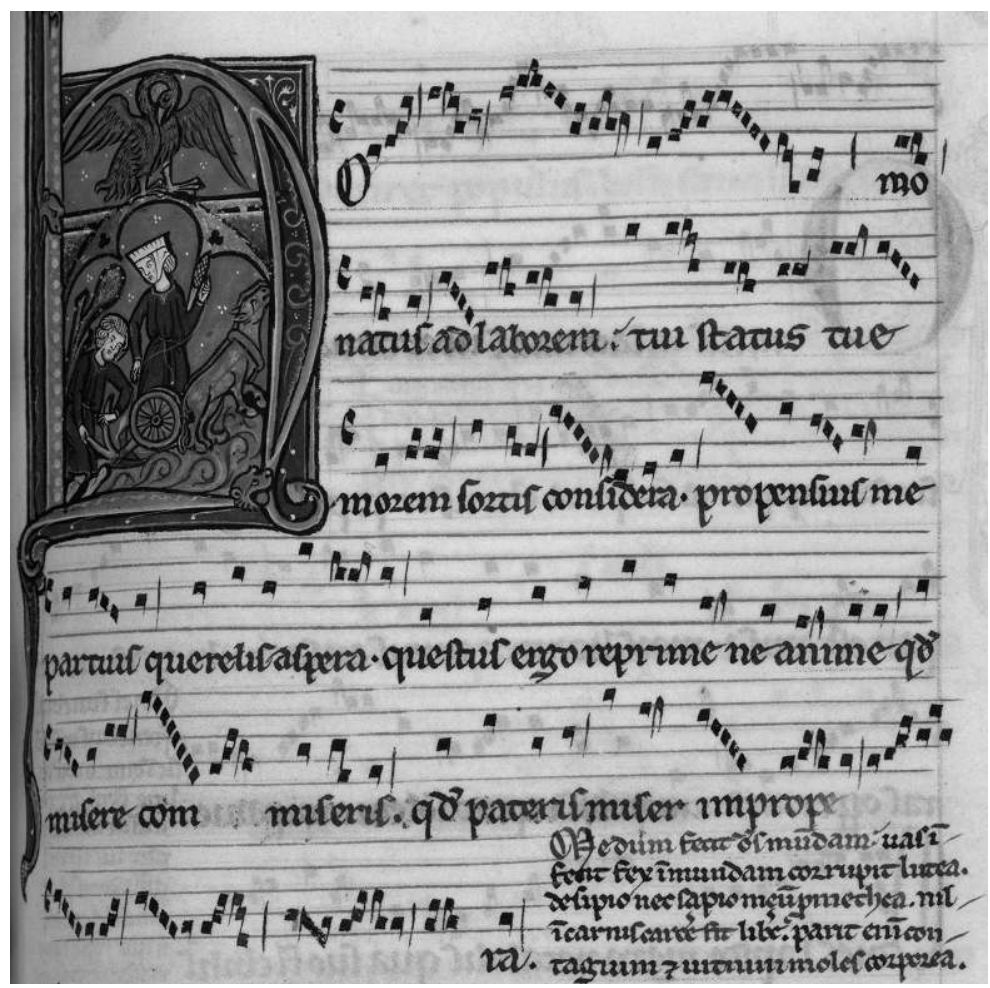

Tel qu'il est présenté dans F, le conduit est de forme assez complexe: les strophes sont doubles (deux textes pour une même mélodie), mais irrégulières (11, 6 et

29. R. BALTZER («Thirteenth Century Illuminated Manuscripts and the Date of the Florence Manuscript», Journal of the American Musicological Society, 25 (1972), p. 1-18) a évoqué la présence de cet oiseau pour supposer une inversion possible avec un autre conduit, lui aussi de Philippe le Chancelier, citant l'intégralité du verset: Homo natus ad laborem / et avis ad volatum (London, Egerton 274, f. 42). Il me semble pourtant que l'oiseau se justifie parfaitement dans ce contexte par la citation biblique et qu'une erreur aussi importante de la part du copiste est fort peu probable à la vue du soin apporté à la réalisation de ce manuscrit. De plus, le conduit choisi pour figurer en exergue du fascicule propose des dimensions et une subtilité que n'a pas le conduit du manuscrit de Londres, unicum de facture plus modeste. 
5 vers). Les deux dernières pourraient n'en former qu'une seule ( $6+5=11$ vers), équivalente à la strophe 1 . Cependant, la composition musicale et la présentation dans le manuscrit séparent délibérément ces deux unités : la présence d'un mélisme à la fin de la strophe 3 , sur la pénultième syllabe de pondere, marque la séparation entre les deux unités de texte. Notons que les sources qui ne rapportent que le texte ne se composent que de deux strophes, les deux dernières n'en formant qu'une ${ }^{30}$. C'est donc la mise en musique qui a rendu audible cette organisation tripartite.

\begin{tabular}{|c|c|c|}
\hline $\begin{array}{c}\text { Strophes } 1 \text { et } 2 \\
11 \text { vers }\end{array}$ & $\begin{array}{c}\text { Strophes } 3 \text { et } 4 \\
6 \text { vers }\end{array}$ & $\begin{array}{c}\text { Strophes } 5 \text { et } 6 \\
5 \text { vers }\end{array}$ \\
\hline $8 a 8 a 6 b 4 c 4 c 6 b 7 d 4 d 8 e 4 e 6 b$ & $8 a 8 a 6 b 4 c 4 c 6 b$ & $7 d 4 d 8 e 4 e 6 b$ \\
\hline
\end{tabular}

Les trois strophes musicales sont ainsi de longueur décroissante, choix original et stratégique instituant un effet d'accélération dans la perception temporelle. La progression de la structure accompagne l'attention de l'auditeur de manière à éviter la lassitude et l'impression de longueur.

De plus, l'utilisation de la forme musicale binaire (deux strophes de texte pour une strophe mélodique répétée) permet une appréhension plus facile du texte, par le truchement de la mémoire immédiate qui reconnaît une mélodie entendue à la strophe précédente. Cette structure n'est pas rare dans les conduits du manuscrit de Florence, mais elle est ici exceptionnellement marquée et appuyée par les jeux de parallélisme du texte ${ }^{31}$. Les strophes se correspondent deux à deux par l'utilisation de mots aux sonorités identiques:

\begin{tabular}{|l|l|}
\hline \multicolumn{1}{|c|}{ Strophe 3} & \multicolumn{1}{c|}{ Strophe 4 } \\
\hline $\begin{array}{l}\text { In abyssum culpe ducis } \\
\text { Que commissum } \text { opus ducis }\end{array}$ & $\begin{array}{l}\text { In abusum rationis } \\
\text { Vertis usum teque bonis }\end{array}$ \\
\hline
\end{tabular}

Figure 2: début de la deuxième strophe musicale

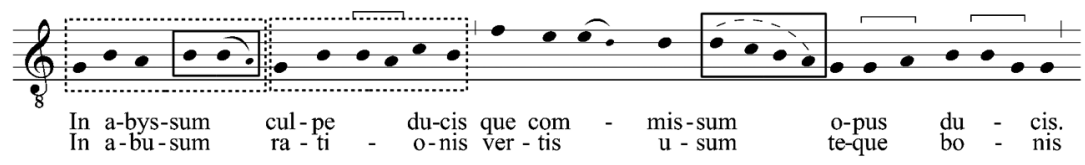

30. Oxford, Add 44, f. 127.

31. Les parallélismes sont tels que certains ont interprété ce conduit comme un dialogue entre le corps et l'âme. Les éditeurs des Analecta Hymnica lui ont donné le titre suivant: Altercatio animae et corporis (AH 21, 115). Il me semble pourtant que ce texte doit être compris comme un monologue de l'âme et non comme un dialogue. 
Le rythme du premier vers (deux quadrisyllabes, en pointillés dans la figure 2) est souligné par la ligne mélodique qui tourne sur elle-même en reprenant un motif simple presque à l'identique. La rime interne dans le deuxième vers (commissum et usum qui font écho, à abyssum et abusum) est accompagnée d'une descente mélodique vers la finale $(s o l)$, préparée dès le début du vers dans l'aigu.

Les deux strophes suivantes commencent elles aussi par une anaphore très marquée: Tibi nomen anime à la strophe 5 et Tibi cogor obsequi à la strophe 6. La musique souligne ces deux pronoms par une marche de tierces dans l'aigu du mode.

Figure 3 : début de la troisième strophe musicale

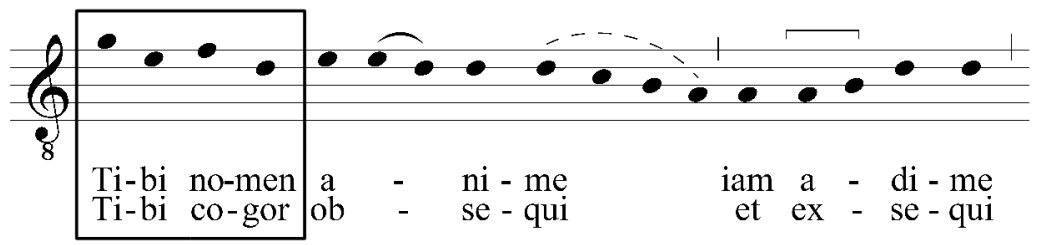

De tels signaux font fonction de marqueurs pour faire entendre le début de la strophe et attirer l'attention sur la structure binaire. Les deux premières strophes (les plus longues) ne présentent apparemment pas de tels parallélismes. Pourtant, il faut remarquer la reproduction d'une figure d'anominatio (paronomase) à la même place, entre les vers 1 et 2, créant un lien subtil entre les deux strophes:

\begin{tabular}{|l|l|}
\hline \multicolumn{1}{|c|}{ Strophe 1 } & \multicolumn{1}{c|}{ Strophe 2 } \\
\hline $\begin{array}{l}\text { Homo natus ad laborem } \\
\text { tui status tue morem } \\
\text { sortis considera. }\end{array}$ & $\begin{array}{l}\text { Me dum fecit Deus mundam } \\
\text { vas infecit fex immundam } \\
\text { corrupit lutea. }\end{array}$ \\
\hline
\end{tabular}

L'organisation d'ensemble du conduit est donc pensée avec les effets sonores qui permettent d'en suivre les subtilités pour en faire un jeu sur lequel s'adosse l'audition. Chaque élément de la structure est signalé par des moyens verbaux (parallélismes d'une strophe à l'autre) ou musicaux. Les mélismes ont, entre autres, fonction d'introduire et de conclure les principaux éléments de la structure. Le premier mélisme assure pleinement sa fonction introductive. Il se compose de deux parties, la première exposant le mode de sol dans sa version authente (où la finale sol est la note la plus grave), la seconde dans sa version plagale (la mélodie se dispose de part et d'autre de la finale).

Figure 4 : mélisme introductif

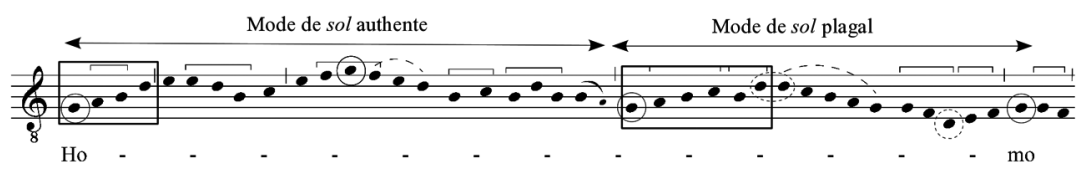


Ces deux phrases mélodiques commencent par un motif ascendant similaire, caractéristique du mode (voir les cadres dans la figure 4). Le mode qui sera celui de toute la pièce est ainsi présenté et l'auditeur se trouve dès le début dans un contexte sonore qui lui est familier. Cette introduction purement musicale est complétée par la citation biblique du livre de Job, comme un repère ou une référence pour l'intelligence. Le poète semble ici suivre un conseil souvent donné dans les manuels de rhétorique depuis l'Antiquité et répété dans les artes poeticae contemporaines, celui d'utiliser une citation ou un proverbe comme matière d'introduction ${ }^{32}$. La citation biblique est isolée de la suite par la mélodie qui achève la phrase sur la finale, en même temps que s'achève la citation, puis reprend dans un registre aigu (tui status). Dans la mise en page du manuscrit de Florence, l'œil est également guidé dans cette entrée en matière par la présence de l'enluminure qui illustre le verset cité.

Regardons maintenant ce qui tient lieu de conclusion à l'ensemble: le mélisme chanté à la fin des deux dernières strophes est composé d'une péroraison de notes répétées dans l'aigu (figure de rhétorique appelée florificatio vocis) et un resserrement progressif sur la finale sol. Le discours se referme ainsi après avoir donné ses derniers éclats :

Figure 5: mélisme conclusif

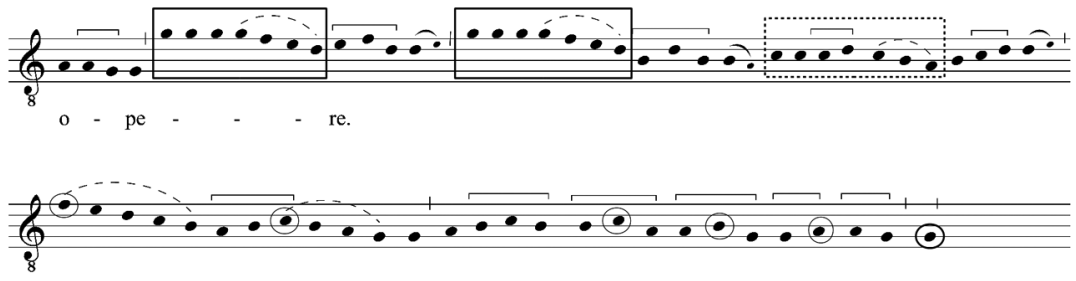

Une autre cauda survient à la fin de la strophe 1, sur le mot impropera. Elle présente elle aussi le même procédé mélodique que le mélisme précédent.

Figure 6: mélisme final de la strophe 1

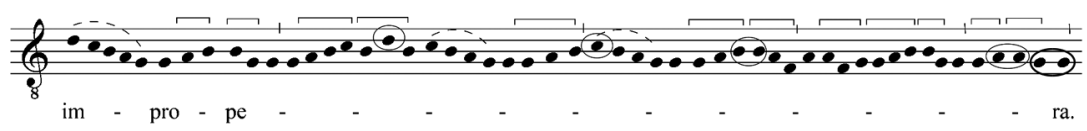

L'auditeur est donc clairement guidé dans sa perception par la mélodie, et la forme d'ensemble se détache comme un cadre clair et rationnel.

32. Voir notamment les exemples donnés par Jean de Garlande (le grammairien) au chapitre 3 de sa Parisiana poetria (éd. T. Lawler, New-Haven - Londres, 1974). 
Chaque double strophe propose une mélodie nouvelle, là aussi selon un parcours progressif de proportions décroissantes, une conduite à travers la variété des propositions mélodiques. La stratégie oratoire est donc ici celle de la diversité, et non celle de la répétition, contrairement à d'autres conduits ${ }^{33}$. Chaque double strophe est organisée comme une entité close, ménageant ses propres effets à son échelle, ses transitions et sa dynamique particulière. Une stratégie d'ensemble élabore des repères temporels régis par des proportions claires à tous les niveaux de la structure. Les sons musicaux (hauteurs, mélismes) et les sons poétiques (figures, répétitions de mots et de syllabes) agissent à la manière de repères visuels sur une page pour organiser l'espace sonore selon les mêmes modalités et schémas qu'un espace graphique. J'ai déjà signalé la fonction introductive de l'enluminure. Un autre détail montre à quel point les éléments convergent pour manifester la structure. La décoration de la lettrine encadre le bloc que constitue la première strophe mélodique. L'auditeur (ou le lecteur ${ }^{34}$ ) est ainsi guidé par les sons pour emmagasiner dans son esprit et sa mémoire les mots qui délivrent un message fort. Cette parole chantée et ce message moral s'adressent à tout un chacun. L'homme désobéit à l'ordre naturel divin en laissant les inclinations du corps prendre le dessus. L'âme qui est le locuteur de ce texte et qui se substitue à la voix du poète, clame son impuissance à maîtriser les abus et les déviances dictés par le corps.

\section{La construction polyphonique au service du Salut}

Notre deuxième exemple est un conduit polyphonique à deux voix (manuscrit F, f. 299). Le texte est composé de trois strophes régulières de sept vers chacune, que le sens, les phrases grammaticales et la versification partagent en un quatrain $(8 a 7 b 8 a 7 b)$ et un tercet $(8 c 8 c 7 d)$. À cette construction régulière s'ajoute une polyphonie différente pour chaque strophe (composition continue). La musique a donc la possibilité de s'adapter aux particularités de chaque entité du texte, ce qui n'est pas le cas d'une composition strophique répétitive. Il est

33. La majorité des conduits monodiques est de forme strophique. La répétition mélodique y est donc systématique. D'autres conduits comme $O$ mens cogita (ms. F, f. 338v-339) sont constitués de strophes doubles, elles-mêmes répétitives. Pour une analyse de ce conduit, voir A.-Z. RILLon, «Convaincre et émouvoir: les conduits monodiques de Philippe le Chancelier, un médium pour la prédication?», dans O. Cullin éd., La Place de la musique dans la culture médiévale, Actes du colloque Singer-Polignac le mercredi 25 octobre 2006, Turnhout, 2007, p. 99-113.

34. L'architecte du ms. F a probablement cherché à reproduire des réflexes liés à son appréhension et sa compréhension de la pièce. La mise en page matérialise la perception. Il faut remarquer que les autres enluminures du ms. F ne s'accordent pas de manière aussi significative à la structure de la pièce présentée. Cet élément semble donc particulièrement prégnant dans la conception que l'on se faisait du conductus simplex. 
intéressant d'observer les variations structurelles apportées par cette mise en forme sonore renouvelée pour chaque partie. Les ponctuations que sont les cadences et les mélismes sont les moyens musicaux employés pour organiser temporellement la perception du texte. La place des mélismes (caudae), indiqués ci-dessous en gras, ainsi que celle des cadences (signalées par le signe /) n'est pas identique pour chaque strophe:

\begin{tabular}{|l|l|l|}
\hline Austro terris / influente. & Innovatur / terra vetus & Serpens dirus / exturbatur \\
surgens cedit aquilo, / & novo more germinans. & ad vagitum pueri. \\
flatu sacro succedente; & parit virgo prodit fetus & per quem pauper liberatur. \\
pulso mentis nubilo ; / & Eve luctum terminans. / & potens datur carceri./ \\
fervet ignis sed fecundans. & quo complente / vis legale; $;$ & ab erroris via flexus; \\
et humanum cor emundans & coaptantur celo scale. & patris redit in amplexus \\
a letali frigore / & via patet regia./ & minor natu filius. / \\
\hline
\end{tabular}

Les cadences principales arrivent sur la finale $(s o l)$ par mouvement contraire des deux voix. Elles permettent de ménager des arrêts dans la continuité du discours et sont un autre moyen que les mélismes pour organiser la strophe par une ponctuation musicale. Les trois strophes marquent l'arrêt entre le quatrain et le tercet. Une autre cadence se trouve régulièrement à la césure du premier vers de chaque strophe, comme pour marquer la fin de l'introduction. Celle-ci, comme la conclusion d'ailleurs, est matérialisée par un mélisme suivi d'une cadence. Le mélisme sur Austro ne surprend donc pas, pas plus que celui sur la deuxième syllabe de frigore à la fin de la strophe 1. On s'interroge davantage sur la raison d'être du mélisme sur pulso, au début du quatrième vers. Ce dernier est plus long, plus orné et plus éloquent que le mélisme introductif qui, pour sa part, est relativement court. Le deuxième mélisme du conduit peut donc avoir pour fonction de surenchérir sur le premier, tout en marquant le parallélisme rythmique entre les deux vers (Austro terris influente / pulso mentis nubilo). Le quatrain initial gagne ainsi en cohérence et peut s'achever sur une consonance d'unisson sur la finale à la fin du vers. 
Figure 7: deux premiers mélismes de la strophe 1

Début du vers 1: $\quad$ cadence

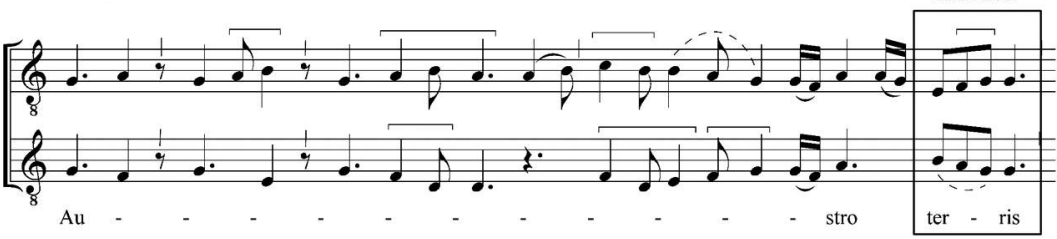

Début du vers 4 :
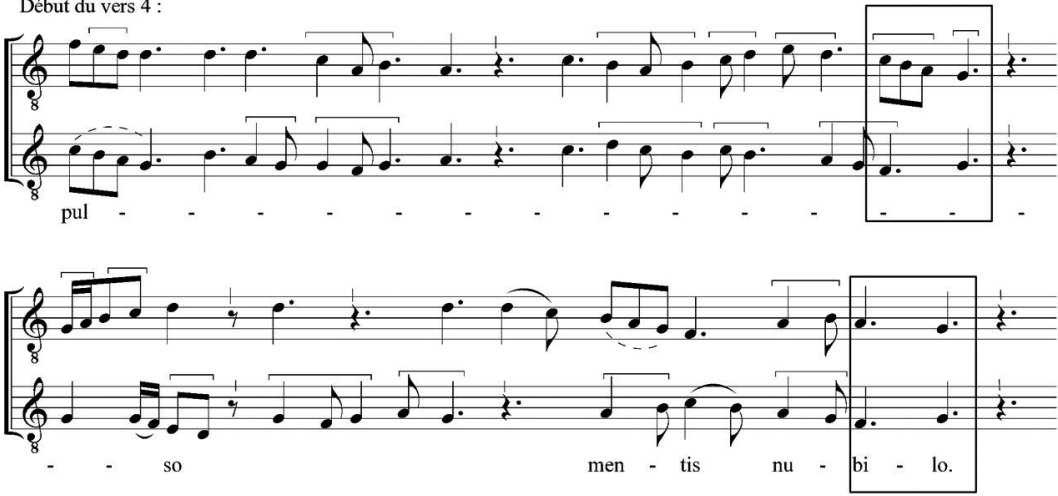

Les deux mélismes qui suivent sur fecundans puis frigore croissent par leur longueur et la richesse de leur ornementation. On y entend des répétitions (colores) ${ }^{35}$ ainsi que des successions de notes rapides descendantes (conjoncturées), procédé mélodique d'ordre rhétorique auquel les deux premiers mélismes n’ont pas fait appel. Les quatre mélismes de la strophe 1 instaurent donc une progression, tant par la taille que par les effets rhétoriques convoqués.

La deuxième strophe ne dispose pas les mélismes de la même manière. Cette fois, le quatrain est divisé en deux groupes de deux vers. Les deux premiers sont délimités par des mélismes (Inovatur et germinans). La place du premier mélisme sur la seconde syllabe (Inovatur) peut s'expliquer par le désir de souligner la paronomase avec le vers suivant (novo more). La suite du quatrain est énoncée de manière plus syllabique car ces vers sont particulièrement importants pour la compréhension de l'ensemble. Le conduit est en effet dédié à la Nativité. Ces deux vers sont les premiers à en parler sans métaphore (parit virgo prodit fetus). Le mélisme sur quo marque le passage à la deuxième partie de la strophe. Il est court et simple, contrairement au dernier qui s'étend sur tout le vers (via

35. R. E. Voogt, Repetition and Structure in the Three- and Four-Part Conductus of the NotreDame School, Ph. D. diss. Ohio State University, 1982. 
patet regia) et propose une construction très élaborée. La dernière strophe est la plus mélismatique de toutes, puisque des caudae s'ajoutent tous les deux vers au moment de la rime: exturbatur, liberatur, flexus et le mélisme final sur filius. Il y a donc eu, depuis le début du conduit, la mise en place d'une surenchère, chaque strophe étant plus mélismatique que la précédente. La stratégie est inverse au conduit Homo natus ad laborem dans lequel nous avions constaté la mise en place de strophes de plus en plus courtes. Les mélismes les plus complexes sont les deux caudae qui terminent les strophes deux et trois. De tels passages présentent leur propre cohérence rhétorique et leur propre progression. Il s'agit d'un discours miniature intégré à l'ensemble. Il n'est donc pas étonnant que le dernier mélisme de ce conduit ait été, par la suite, séparé du corps du conduit pour devenir une composition indépendante. Chaque note a été pourvue d'une syllabe (procédé du trope). Le nouveau texte reprend le dernier vers minor natu filius et développe la parabole du fils prodigue ${ }^{36}$.

Figure 8: manuscrit F, fo 299 (détail)

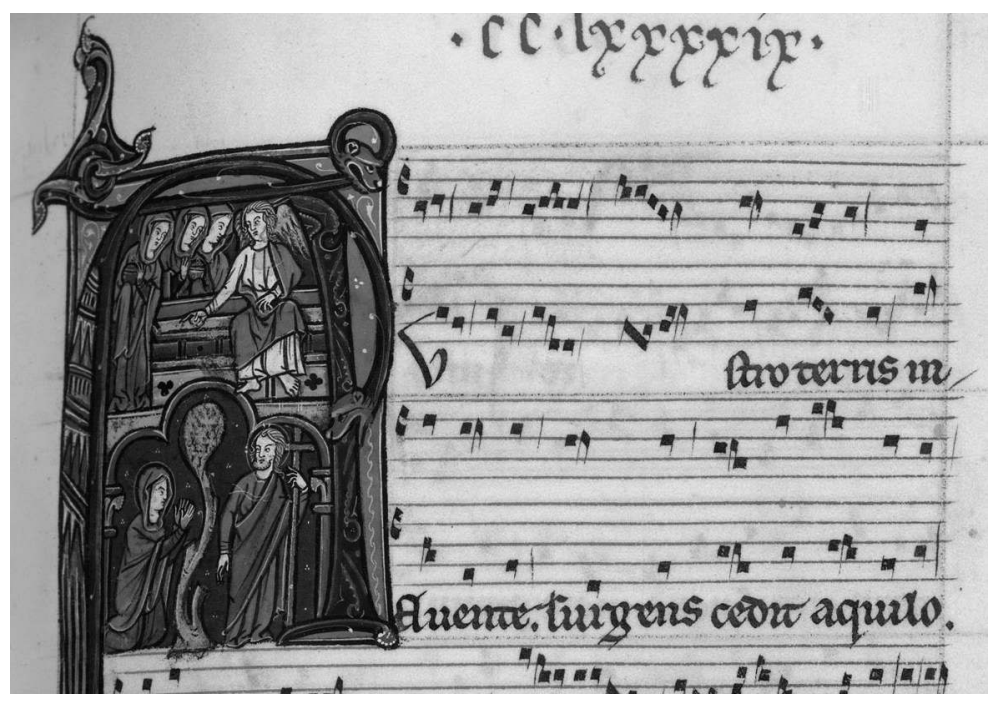

36. Les relations qu'entretiennent le conduit et sa prosule ne sont pas explicites. La prosule est notée dans le même manuscrit parmi les conduits monodiques. On peut se demander si elle était interprétée à une ou deux voix et si son existence était complètement distincte de son conduit d'origine. 
La lettrine du manuscrit de Florence, au folio 299, présente deux parties: dans le haut de la lettrine on reconnaît la scène des trois Marie découvrant le tombeau vide. Dans la partie inférieure, le Christ ressuscité et Marie-Madeleine sont représentés dans un jardin ${ }^{37}$. C'est donc à Pâques et à la Résurrection que l'illustration renvoie directement, tout comme le début de la première strophe du conduit: le message du retour du Christ comme un souffle printanier chasse les rigueurs du froid et purifie les hommes. Ce n'est pourtant pas le thème principal du conduit puisque celui-ci est consacré à la Nativité. Cependant, l'enfantement de la Vierge est bien l'acte originel par lequel la purification commence. La troisième strophe s'achève sur l'évocation de la parabole du fils prodigue, donc sur l'exemple de conversion montré par cet enfant revenu et pardonné par son père, figuration du Christ montrant la voie du Salut aux hommes par sa Résurrection. Le chemin de rédemption ouvert par le Christ est celui que doivent suivre les pécheurs. L'image de ce trajet ascensionnel qui conduit vers le Salut est évoquée par un vocabulaire explicite à la fin de la strophe 2: celo scale, via regia. Il est matérialisé par ces trois strophes et cette illustration qui donnent à voir et entendre différentes images du mystère du Salut.

Dans ces deux exemples, comme dans bien d'autres que l'on trouve à leurs côtés dans les sources du XIII ${ }^{\mathrm{e}}$ siècle, la composition poétique et musicale met en place diverses stratégies sonores afin de montrer la voie du Salut et expliquer le message du Christ, le seul véritable guide. Le terme conductus démultiplie ainsi ses applications et ses acceptions. Son étymologie ne fait plus référence à sa fonction, mais à son contenu, et aussi, devrait-on dire, au pouvoir de son contenant, si l'on peut considérer ainsi la stratégie élaborée dans le temps au moyen des mots et du chant. Il s'agit de montrer le chemin à parcourir, et la conduite à adopter. Mais, en plus de montrer la voie, la composition a pour ambition, d'une part, de guider l'auditeur dans son interprétation du mystère pour qu'il trouve lui-même son Salut et, d'autre part, d'être elle-même un instrument agissant pour l'amélioration de l'homme. Les sons et les structures organisent un parcours intelligible, temporel, fait de repères qui agissent comme des images ou loci aidant à construire les représentations de la mémoire. Le temps est découpé en sections hiérarchisées et organisées à la manière d'un espace purement mental. C'est dans ce travail de disposition que se trouve la stratégie ${ }^{38}$. Le déplacement

37. On peut aussi voir un lien entre l'imagerie du Christ-jardinier et le verset du Cantique des Cantiques $(4,16)$ dont s'inspire l'incipit du conduit: Surge aquilo et veni auster. Perfla hortum meum et fluant aromata illius.

38. Nancy von Deusen arrive à des conclusions approximativement similaires en partant d'autres éléments pour sa démonstration. Dans deux études («Figura, Ductus, Conductus: Thirteenth-Century Discussions of Rhythm in Context», The Harp and the Soul Studies in Early Medieval Music and 
physique qui a donné son nom à des œuvres plus anciennes avait déjà un rôle éminemment symbolique dans l'espace réel qu'est l'église : l'accompagnement du chant et du texte peut être interprété comme une élévation spirituelle qui fait redondance avec l'action effectuée et amplifie son effet. Une fois dépourvu de son usage pratique liturgique, le conduit n'en reste pas moins un guide entièrement transposé dans le domaine spirituel, purement auditif et intellectuel. Ce rôle peut encore se lire dans la rime riche qu'Henri d'Andeli exploite dans le Dit du Chancelier Philippe. À la Vierge pour laquelle Philippe a composé de nombreuses chansons et conduits, le trouvère normand s'adresse en ces termes:

Virge rö̈ne coronée,

Se met del tot en ton conduit,

Car il fist de toi maint conduit ${ }^{39}$.

Pourtant, l'utilisation du mot conductus dans le vocabulaire courant des $\mathrm{XII}^{\mathrm{e}}$ et $\mathrm{XIII}^{\mathrm{e}}$ siècles semble éloigné de la sphère du spirituel. Dans les Écritures, le conductum est utilisé dans un sens commercial ${ }^{40}$ : il est une location, participe qui dérive de l'un des sens de conducere, louer (Gaffiot). À l'époque féodale, le terme s'est élargi et enrichi de nombreux sens : le conductus (quatrième déclinaison) est une escorte pour les voyageurs. Par extension, il désigne le transport, la protection due aux voyageurs et aux marchandises, les protégés (les hommes, le ravitaillement et même la nourriture) et les protecteurs (les compagnons armés) ou encore la rétribution, le salaire ou les frais de rétribution pour ces déplacements. Niermeyer recense onze sens ${ }^{41}$. La onzième entrée pour conductus est «chant d'accueil » et fait référence à nos compositions, sans expliquer le lien avec le groupe sémantique large, mais cohérent, des dix entrées qui précèdent. Pourtant, en analysant comme nous l'avons fait les compositions désignées par ce terme, on peut comprendre comment un poème mis en musique peut escorter, guider, sécuriser les esprits en quête d'espérance, mais aussi en être la nourriture, comme un ravitaillement pour la conscience.

its Role in the Intellectual Climate of the Early University, New York, 1989, p. 307-328 et «On the Usefullness of Music: Motion, Music, and the Thirteenth-Century Reception of Aristotle's Physic », Viator, 29 (1998), p. 167-187), l'auteur montre comment la terminologie musicale, notamment le terme conductus, se nourrit de la pensée aristotélicienne et de sa réception par les penseurs universitaires et les théoriciens de la musique de la seconde moitié du xIII ${ }^{\mathrm{e}}$ siècle. Cette interprétation ne tient pas compte du fait que le terme conductus désignait des compositions musicales bien avant que la pensée aristotélicienne ne fût diffusée dans les milieux scolaires.

39. Les Dits d'Henri d'Andeli, v. 140-142.

40. Exode 22, 15: «quod si inpraesentiarum fuit dominus non restituet maxime si conductum venerat pro mercede operis sui.»

41. J.F. NIERMEYER, Mediae latinitatis lexicon minus, Leyde, 1976, p. 241-242. 
La conduite de l'esprit... c'est d'ailleurs le sens du ductus rhétorique tel qu'il est défini par les anciens et repris par les médiévaux. Selon Mary Carruthers, «le ductus représente [donc] la manière dont une composition donnée guide quelqu'un jusqu' aux buts qu'elle s'assigne ${ }^{42}$. Le ductus du conductus est donc sonore dans tous les sens du terme. Il est la construction d'un espace-temps mental dans lequel chacun trouve et forge ses repères, ses lieux et ses images au moyen des sons et des rythmes disposés par le poète, jongleur de Dieu.

\section{Annexes : textes de Homo natus ad laborem et Austro terris influente ${ }^{43}$}

1.

Homo natus ad laborem; tui status tue morem sortis considera. propensius me parcius querelis aspera. questus ergo reprime ne anime quod misere commiseris. quod pateris miser impropera.

3.

In abyssum culpe ducis que commissum opus ducis. procuras temere me perimis cum opprimis peccati pondere.

5.

Tibi nomen anime jam adime quia recte non animas cum perimas me mortis opere.
2.

Me dum fecit Deus mundam; vas infecit fex immundam corrupit lutea. desipio nec sapio meum Promethea. nil in carnis carcere fit libere. parit enim contagium et vitium moles corporea.

4.

In abusum rationis vertis usum. teque bonis privas gratuitis. dum sensibus assensibus faves illicitis.

6.

Tibi cogor obsequi et exsequi opus rectum si judices. vel claudices a recti semitis.

42. M. CARruthers, The Craft of Thought, Meditation, Rhetoric, and the Making of Images, Cambridge, 1998; Paris, 2002 pour la traduction française, p. 104-109; voir aussi «The Concept of Ductus, or Journeying through a Work of Art», dans EAD. éd., Rhetoric Beyond Words, Delight and Persuasion in the Arts of the Middle Ages, Cambridge, 2010, p. 190-213.

43. Les textes sont donnés d'après le manuscrit de Florence, sans ajout de ponctuation. 
1.

Austro terris influente. surgens cedit aquilo.

flatu sacro succedente; pulso mentis nubilo; fervet ignis sed fecundans. et humanum cor emundans a letali frigore.

2.

Innovatur terra vetus novo more germinans. parit virgo. prodit fetus Eve luctum terminans. quo complente vis legale; coaptantur celo scale. via patet regia.

3.

Serpens dirus exturbatur ad vagitum pueri. per quem pauper liberatur. potens datur carceri. ab erroris via flexus; patris redit in amplexus minor natu filius.

Anne-Zoé Rillon-Marne - CESCM, 24, rue de la Chaîne, F-86022 Poitiers

Stratégies pour la conduite des âmes : la composition poétique et musicale des conductus parisiens au début du XIII ${ }^{\mathrm{e}}$ siècle

Dès son apparition au début du XII ${ }^{\mathrm{e}}$ siècle, le conductus s'affirme comme une pratique musicale liée à l'espace. Bien que les données manuscrites ne soient pas toujours claires, le terme conductus apparaît dans les sources musicales et désigne les pièces ajoutées à la liturgie accompagnant certains déplacements et processions ou servant d'introduction aux lectures. Cette dernière fonction montre déjà une tendance à utiliser le terme de «conduit» dans un sens figuré. Pendant le $\mathrm{XIII}^{\mathrm{e}}$ siècle, le terme est utilisé pour désigner une partie du répertoire des compositions latines, les conduits dits «de Notre-Dame». Ceux-ci sont désormais plus faiblement connectés à la liturgie que ne l'étaient ceux des répertoires antérieurs. Nous cherchons donc à comprendre en quelle mesure le terme utilisé au XIII ${ }^{\mathrm{e}}$ siècle reste pertinent pour désigner le nouveau répertoire. 
En choisissant deux exemples de conductus dans le corpus poético-musical attribué au théologien, prédicateur et poète-compositeur Philippe le Chancelier, nous proposons de montrer comment comprendre le sens du terme conductus dans le contexte parisien. Par la combinaison des moyens de la poésie rythmique et de la mélodie, ses deux œuvres (Homo natus ad laborem et Austro terris influente) sont savamment élaborées pour guider l'auditeur dans une démarche de réflexion, pour le «conduire», par les sons et les images (poétiques et visuelles), à construire sa pensée. Le pouvoir du conductus se manifeste ici par des jeux de structures, des figures poétiques et musicales (colores), des références et des citations qui sont autant de repères pour l'oreille et l'intelligence d'un public averti. De sa fonction originelle d'accompagnement, le conductus conserve son lien à l'espace, mais celui-ci est désormais mental et permet aux auditeurs de construire leur chemin vers le Salut.

Musique - poésie - Paris - conductus - Philippe le Chancelier.

\section{Strategies for the Conduct of Souls : the Poetic and Musical Composition of the Early xiIth Century Parisian Conductus}

From its beginnings in the early twelfth century, conductus is clearly a musical practice related to space. Although manuscript informations are not always easy to understand, the word « conductus » appears in musical sources and designates some pieces added to liturgy to accompany certain movements and processions or as an introduction to the readings. This latter function shows an early tendency to use the term «conductus » in a figurative sense. During the thirteenth century, the term is used to designate a part of the Latin repertoire called «Notre-Dame conductus ». These are now less connected to liturgy than they used to. We seek to understand how the term used in the thirteenth century is still relevant to describe the new corpus.

By choosing two conductus in the poetic-musical corpus attributed to the theologian, preacher, poet and composer Philip the Chancellor, we propose to show how to understand the meaning of conductus in the Parisian context. By combining the resources of poetic rhythm and melody, his two works (Homo natus ad laborem and Austro terris influente) are cleverly designed to guide the listener through a process of reflection and to «conduct» him by the sounds and images (visual and poetic), to construct his thought. The power of conductus is manifested here by the interplay of structures, poetic and musical figures (colores), references and quotations that are cues for ear and intelligence of the audience. From its original accompanying function, the conductus retains its connection to space, but it is now a mental space that allows listeners to build their own way to Salvation.

Music - poetry - Paris - conductus - Philippe the Chancellor. 